\title{
EAI Endorsed Transactions

\section{How annotated visualizations in self-care technology supported a stroke survivor in goal setting and reflection}

\author{
B.I. Hougaard and H. Knoche \\ Aalborg University, Rendsburggade 14, 9000 Aalborg, Denmark
}

\section{Abstract}

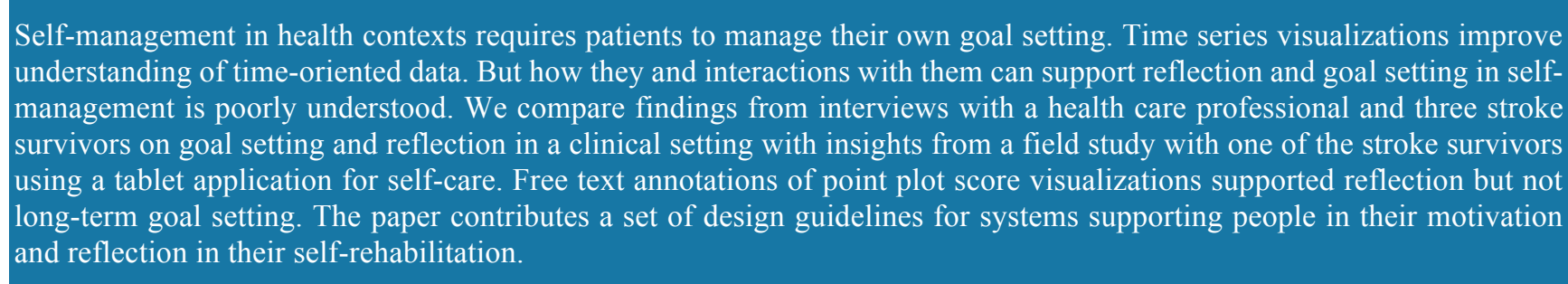

Keywords: self-care technology, rehabilitation, visualization, reflection, goal setting, stroke victims, trail making test

Received on 18 October 2017, accepted on 29 November 2017, published on 8 December 2017

Copyright (C) 2017 B.I. Hougaard and H. Knoche, licensed to EAI. This is an open access article distributed under the terms of the Creative Commons Attribution licence (http://creativecommons.org/licenses/by/3.0/), which permits unlimited use, distribution and reproduction in any medium so long as the original work is properly cited.

doi: 10.4108/eai.8-12-2017.153400

\section{Introduction}

Aging societies are facing challenges to curb healthcare costs especially for rehabilitation, which becomes more common in aging populations. Patient empowerment constitutes a promising avenue towards increasing efficiency and effectiveness of healthcare provisioning by enabling patients to become a more active and motivated partner often in conjunction with selfcare technology. For example, blood glucose meters support diabetes patients in selfmanagement. Goal setting constitutes an important factor in rehabilitation efforts and a common concern in self-management for motivation purposes.

Health care professionals include patients in patient-centered goal setting through an informal discussion on how and when to carry out

${ }^{*}$ Corresponding author. Email: hougaard.junior@gmail.com rehabilitation activities [20]. Insights from reflecting on their own conditions and changes thereof, empower patients in HCP interactions and are crucial for self-management where patients lack HCP availability.

Within self-care technology and other domains, information visualization enables reflection by making trends and patterns visible [1,2] and allowing for tracking progress and comparison with goals [15][14]. Time series visualizations represent the most common format for timeoriented data typical for self-care technology $[1,2]$.

However, little research has addressed how visualizations of temporally accrued quantitative data and interactions with them address user needs in for reflection and goal setting in selfcare settings at home and how different stakeholders such as friends, family, and health care professionals affect them. 
Serious games provide a means of creating selfcare technology with engaging experience from which to obtain quantitative data, for visualizing progress and goal setting.

The paper relies on a self-care technology case study of a stroke patient tracking Trail Making Test (TMT) [5] data through a serious game for insights on a) how clinical environments and HCPs supported stroke patients in goal setting in a rehabilitation centre and b) how information visualization supported one patient's needs in self-management and goal setting after discharge. The paper concludes with a set of design guidelines on interactions with visualization formats for self-care settings.

\section{Background}

Stroke survivors can recover lost abilities by undergoing rehabilitation during which health care professionals assist in targeted goal setting and motivation to organize daily cognitive and physical training activities [21]. Setting goals jointly with clinical therapists increases patients' involvement in the rehabilitation process [24]. Making progress towards personally motivated goals improves patients' self-image and constitutes a coping mechanism [21].

After discharge and rehabilitative regimens end, stroke survivors need to be able to self-manage their chronic condition and recovery including symptoms, treatment, psycho-social consequence, and life style changes [4]. But at that point the goal setting and motivation is left to patients and their social networks with little or no support from HCPs.

In health related and self-care settings, people self-track, i.e., log data about themselves for four reasons: documentation vis-a-vis their health care providers [3], communication with e.g. family members [16], generally to improve wellbeing [8-10], or for reflection, selfunderstanding, and advice $[1,8,9]$.

When people reflect on tracked data, they can be either in the discovery phase (in which they identify goals to meet and exploring what factors influence them) or maintenance phase (in which they compare current status with their goals to monitor progress) [14].
Fleck \& Fitzpatrick identified different 'levels of reflection' of activities and behaviours [8]. These include reflective descriptions $(\mathrm{R} 1)$ that explain in a reportive or descriptive way, dialogic reflections (R2) that see things from different perspectives and try to identify relationships, transformative reflections (R3) that change the original point of view due to gained knowledge, and critical reflections $(\mathrm{R} 4)$ that include a wider perspective beyond the immediate context.

Rivera-Pelayo et al. identified commonly tracked dimensions to reflect (e.g. emotional aspects, and the social, spatial, and historical context) [20].

Interactions with information visualizations are equally important for enabling reflection [22]. Current research on interactions with visualizations focus on generic techniques such as selecting or filtering [7] but not on how these techniques relate to contextual parameters or address concrete needs for higher level tasks such as reflection.

Annotations can facilitate reflection and selfefficacy $[9,23]$ e.g. when people collect and organize information through annotations [23]. Webb et al. referred to this as reflection-incuration, a subset of Schön's reflection-in-action (reflection surfacing through doing) [22]. Tracking data over time is central to goal setting, tracking progress, and reflection. Since medieval times, line plot visualizations have depicted timeoriented data allowing for reviewing history, discovering developments, and exploring patterns [2].

Research on time series visualization has focused on graphical perception and evaluating novel ways of visualizing data [1,2]. For example, people were more effective and confident when visualizations represented time series data in a Cartesian compared to a polar coordinate system in Adnan's study [1]. Interaction techniques, e.g. highlighting of data points and using tooltip techniques for value identification, improved user experience without decreasing task accuracy or efficiency. Tasks included finding extreme values, comparison of data points, and trend detection [1]. In rehabilitation, serious games provide a means to engage a patient's attention and use player data to visualize feedback of progress and goals [19]. This becomes a means 
for reflection when no HCP is involved. However, research has not addressed how specifically visual representations and interactions with them enable people to reflect on their tracked data for example for goal setting and tracking progress towards them in selfrehabilitation.

To address this, we studied a stroke patients' needs regarding goal setting in a selfmanagement setting by using a time series representation to facilitate self-reflection. The studies' main focus is on cognitive training and takes point of entry in patients' self-managed training of attention using a digital tablet game called TRAILiT, and a grading system based on the Trail Making Test depicted in Figure 1.

\section{Design}

TRAILiT, a digital tablet app version of the Trail Making Test [5], has people visually search for and connect numbers or letters in a specific sequence. The app used visual and audio feedback when circles were connected correctly or incorrectly. After completion of one series of circles (level), TRAILiT congratulated, depicted the current training session duration, and allowed for continuation to the next level. TRAILiT progressed through levels increasing in difficulty

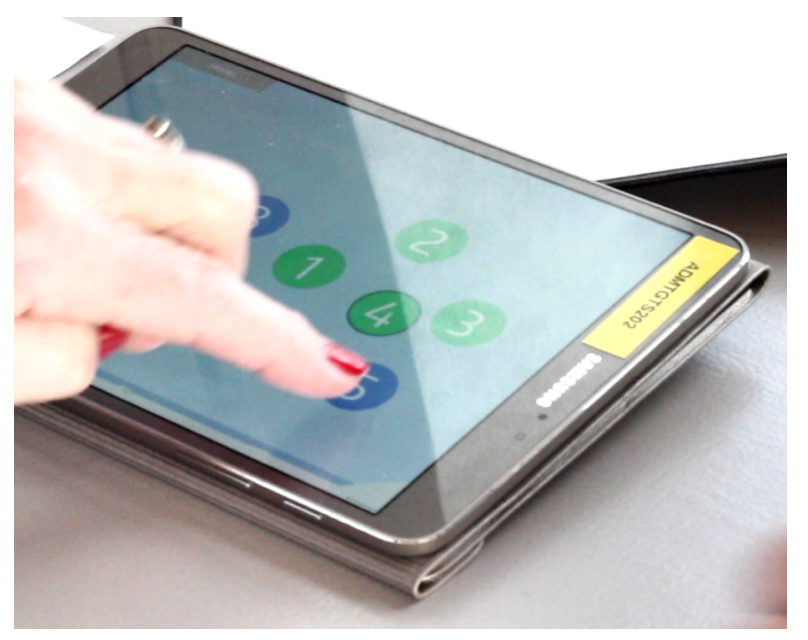

Figure 1. With TRAILiT (left) players connect numbered circles in a certain order to train their cognitive abilities such as attention. The timeline excerpt (middle) plots performance with colour and number until eight minutes had passed. It announced the end of the training session but allowed for voluntary continuation. Players could chose to play levels again from an overview screen which gamified the use by unlocking higher levels and by labelling solved levels with one to three stars according to the player's performance.

\section{Case study - Goal setting in clinical context}

Our case study investigated a stroke patient's experience of undergoing rehabilitation and how artefacts and activities supported the patient in goal setting prior to discharge.

To this end, we interviewed the discharged stroke survivor in her home and her former occupational therapist at the clinic. Paula, a 62-year-old woman with a mathematical background had experienced a stroke and undergone rehabilitation sixteen months prior. During her rehabilitation stay she had played TRAILiT, with a recommended regimen of three times daily for a period of 30 days. In a prior clinical study on self-rehabilitation, she was among the motivated participants and open to using self-managing tools without HCP support. To improve recall, she played an eight minute session of TRAILiT. Afterwards the interview focused on her experience during rehabilitation including a review of her TRAILiT performance 16 months ago. In collaboration with her, the facilitator aligned this data as a timeline with major milestones she recalled from her rehabilitation stay.

The collected data was triangulated and transcribed using the meaning condensation method [12] and further coded and analyzed following a process akin to Yin's Five-Phase Cycle [25].

\subsection{Results}

The patients shared rooms and common areas with other patients during rehabilitation and those peers acted as conversational partners or competitors in game performance. Relatives paid the patients visits and not only gave encouragement in support for the patients' 
motivation but sometimes created tensions as a result of the patients' condition or absence from home.

According to the therapist patients had problems articulating goals based on their long-term desires in rehabilitation. Therapists helped patients to define goals related to life at home achievable in a one to two-week window. To that end, therapists supported patients in weekly goal meetings and arranged a large part of the patient's training activities in the rehabilitation period. On patient request, relatives could participate in these goal meetings. Its dialogue provided a forum for reflection and was an evaluation of past goals with possible addition of new goals and produced a small number (4-5) of concrete and achievable goals. For example, Paula had the goal to "Lift a pot with 20 potatoes and water while walking two meters" and "To read 15 sentences in two minutes". A whiteboard held the list of goals visible to the patient, visitors, therapists, and other patients. Time permitting, HCPs checked off attained goals, to facilitate self-tracking, reflection, and motivation. The patients and HCPs did not use TRAILiT for goal setting purposes.

TRAILiT provided limited information on training progress and goal setting creating a barrier for reflection for the patients who did not become aware of the progress made on a day-today basis. The therapist saw progress indication to benefit patients' motivation through competition with themselves and others. Paula sought level completion times, counts of the connected circles, and the average time to connect a circle as ways of measuring progress in TRAILiT.

\subsection{Discussion}

The distinction between rehabilitation activities and goal setting meetings are very similar to Li's discovery and maintenance phase. The weekly goal meetings provided opportunity for the patient to formulate new goals based on informal discussion with HCPs. Patients could then track their progress (maintenance phase) based on HCP feedback in training activities, and through check marks on the goal whiteboard.
The goal whiteboard acted as progress indication and its high visibility provided opportunity for encouragement, conversations around rehabilitation and reflection alone and with others. The patients' lack of awareness regarding their own short-term progress in rehabilitation suggests that patients might benefit from additional self-monitoring to keep patient involved in training. In the context of attention training, self-monitoring can be provided by TrailIT through performance indications between each level and after each training session.

\section{Home Context}

Inspired by a participatory design approach, we investigated how time series visualization can support conversations and reflections on progress and goal setting in a self-management context that lacks continuous HCP support. Paula did not see herself as a stroke patient anymore.

\subsection{TRAILiT Development}

To help with assessing progress, a re-designed version of TrailIT displayed level completion time, the average time spent in connecting two circles, and the number of mistakes made after each completed level. After session completion, it displayed the total number of circles connected. It resumed and stayed at the same difficulty level throughout a session and the entire study. For each level, it chose one of five fixed circle layouts and shuffled the labels.

\subsection{Method}

A first workshop explored needs and goal setting around visualizing progress and annotating time series visualizations on an A2 paper with contextual data of TrailIT sessions from two years prior in rehabilitation. The visualization featured a classical point plot visualization, with horizontal axis mapped to time segmented by day and vertical axis mapped to total number of circles connected per training session. The facilitator aligned this data with the major rehabilitation events (admission, discharge), milestones, and reached goals. 
During 14 days, Paula trained with TRAILiT three times a day and used two sheets of A2 paper with grids to $\log$ and annotate her performance.

A concluding interview focused on her experience using TRAILiT in conjunction with the performance visualizations, insights from this activity, and her needs for self-management.

\subsection{Results}

Paula trained daily with TRAILiT - 35 out of 42 possible sessions. She tracked and annotated her performance on the timeline visualization by using colored data points (color annotation) using orange, green, and purple for morning, afternoon, and evening sessions respectively (see
Figure 2 right, top). She added the exact number of circles she had connected (number annotation) to the data point. Outside the time series visualization, she used text annotations consisting of a date, often a time, and a short comment (see Figure 2 right, bottom). She created these text annotations during the first seven days. Mentally she preferred to track her fastest level completion time as it facilitated selfcompetition. She did not set explicit goals, e.g. a specific score to attain as it did not come to her mind but she used the current high score as a moving target to surpass.

Paula's text annotations typically were reflective descriptions (R1) consisting of justifications for her performance (such as energy level, her

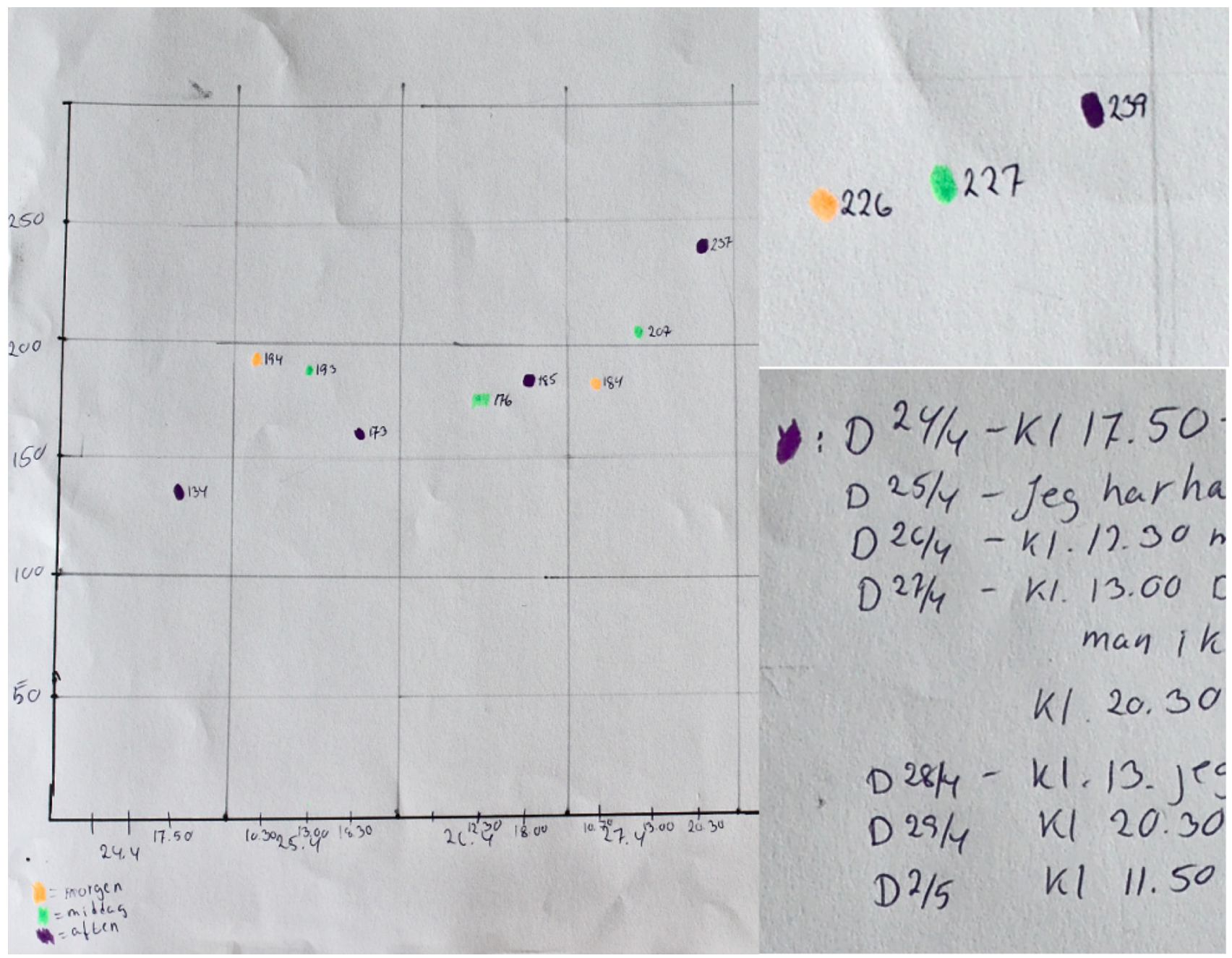

Figure 2. The timeline excerpt (left) plots performance on the vertical axis against time on the horizontal axis. Colour and number annotations (examples on right, top) depicted each training session and time of day and were enriched with separate textual annotations (right, bottom). 
emotions, and other important activities e.g. walking the dog) but with no further exploration of other explanations. She preferred these texts to be short.

Initially, Paula had believed that time of day influenced her performance. But after 14 days of training the colour and text annotations had helped Paula find another explanation (R3 reflection) and she concluded that walking with the dog before training yielded better performance. One large trend, however, went unnoticed. Only when prompted and shown did Paula understand that during the 14 days she had substantially improved her performance.

She kept the time-line visualization out of sight except when logging and annotating data. Paula occasionally had guests and relatives over but chose not to share the time-line, explaining that she found it "too technical" and that some visitors were not aware of her rehabilitation. To them she mentioned the game and her current completion time high score. For daily sharing with peers, Paula preferred a higher level visualization with aggregated results and sharing contexts and keeping "technical" visualizations (e.g. the timeline) for private use or for conversations with HCPs.

\subsection{Discussion}

In the clinical setting the goal whiteboard enabled progress monitoring and enabled visitors to give encouragement. But in the home setting Paula did not want to display her progress, as she did not want to be perceived as a patient or as someone sick anymore. Yet, the peer encouragement was still important for her in the home setting for motivation and she invited this by mentioning the game and her high score to others.

The results suggest that progress indication can sustain training through self-competition. Paula did not set explicit goals around her training progress but TRAILiT's level completion feedback enabled her to mentally track and set implicit goals of beating her best completion time. $\mathrm{Li}$ et al. referred to this activity as shortterm reflection [13].
Time series visualizations provide accurate depictions of progress and create opportunity for discussion between patient and HCP or similar experts around trends, peaks, and patterns. However, without HCP support and goal meetings, self-management contexts lack and need to support the distinction between discovery and maintenance phase as Paula did not engage in longer term goal setting on her own accord. She did, however, keep the implicit goal of beating her current high score. When on her own, the application and the time-series visualization provided her little hands-on support or scaffolding for the discovery of concrete goals that could be attained over time. Continuously annotating data in a time series visualization was not sufficient for the facilitation of such goal setting. While we did not formally test Paula's graph reading ability her mathematical knowledge makes graph illiteracy [18] an unlikely explanation.

Providing long-term predictions or trend lines of performance may be a way to support this [10] but would require future validation in this context.

Paula's reluctance to share the time series visualization with visitors may stem from its level of detail, making it inappropriately time demanding or challenging for others to interpret or the risk of exposing her prior medical condition or incompetence [21]. Using aggregated results addressed Paula's privacy concerns and enabled sharing of progress vital for receiving encouragement and support, as we found in the clinical context. While in clinical contexts patients receive visits from people close to them they might have people less close to them paying visits at home. People self-managing their health and well-being need control over sharing information with these different groups and health care professionals. Paula deemed the timeseries visualizations more appropriate for the latter.

For easier sharing the training session performance unit may benefit from being expressed as completion time, which served as Paula's high score and was clearer than number of connected circles a TRAILiT-specific measure that might require additional explanation. 
Based on the conducted studies we derived a set of design guidelines for self-management systems focused on rehabilitation. These systems should:

- facilitate a distinct discovery phase for goal setting to facilitate reflection for periodical goal setting, e.g. on a weekly basis,

- provide performance feedback whilst training to facilitate self-competition,

- provide aggregated results, which can be shared and easily digested by using relative or other common measurement units,

- allow result annotation with concise contextual data in free text form to facilitate higher levels of reflection,

- provide evaluation of past goals and help with suggesting new goals, e.g. from modelbased predictions from the historical data during discovery phase, and

- allow access to detailed visualization showing current progress, contextual data, rehabilitation goals, targets and reached goals (to support a gamification progress effect), which can be used privately or in discussions with HCPs.

In terms of limitations of our work, the reflections we identified, stem from the interview and the paper artefacts not from a purely digital system. We interpolated from a hybrid digital plus paper approach to the digital domain and assume that potential hurdles e.g. from cumbersome text entry on mobile devices would not place a barrier to text annotations in this health domain setting.

Another limitation was the participant whom we selected because she showed initiative both in the clinical and the home context to engage in selfrehabilitation. Considering findings from e.g. [11] it seems likely that providing patients with a training application outside a service with e.g. HCP involvement will not work for all. Future research needs to focus on whether and how people can be motivated to self-manage their health and which part technology can play. It has to address whether people consider other explanations at the time of logging, annotating, and reflecting whether this can have any transformative impact. In our case, Paula stopped adding free text annotations to her time series after seven days and it remained unclear how reflection and motivation would be affected in real long-term usage.

\section{Conclusion}

Self-management contexts require patient reflection for goal setting and motivation in training activities for which information visualizations provide a promising avenue. We investigated goal setting in clinical context and found that patient goal setting in rehabilitation was supported by HCPs through weekly goal meetings and a goal list indicating progress to the patient, other patients, and visiting relatives who provided encouragement.

An individual case study evaluated how to support stroke patients in a self-care setting at home using progress indication in a serious game TRAILiT and annotation on a time series visualization of the player data. A discharged stroke survivor who trained for 14 days showed that level completion time enabled patient selfcompetition and that text annotations triggered self-reflection. However, the time series visualization was not a suitable medium for peer sharing. The study points towards a patient need for visualization which use aggregated results, e.g. current or evolution of high scores to address privacy concerns and enabling support from peers, as seen in the clinical context.

We presented design guidelines that can help future designs of systems aimed at selfmanagement in the health domain targeting selfreflection to facilitate goal setting. We hope that the case study encourages more work on how information visualization can benefit people selfmanaging their health.

\section{Acknowledgements.}

This work was partially supported by Ideklinikken and Patient@Home.

\section{References}

[1] Adnan, M., Just, M., and Baillie, L. (2016) Investigating Time Series Visualisations to Improve the User Experience. In Proceedings of the 2016 CHI Conference on Human 
Factors in Computing Systems, USA, May 2016 (New York, NY, USA: ACM), pages 5444-5455.

[2] Aigner W., Schumann H., Tominski C,. SPringerLinK, and Miksch S. (2011) Visualization of Time-Oriented Data (London : Springer-Verlag London Limited).

[3] Ancker J. S., Witteman H. O., Hafeez B., Provencher T., GraAf M., and Wei E. (2015) "You Get Reminded You're a Sick Person": Personal Data Tracking and Patients With Multiple Chronic Conditions. Journal of medical Internet research 17(8), e202.

[4] Barlow J., Wright C., Sheasby J., Turner A., and Hainsworth J. (2002) Self-management approaches for people with chronic conditions: a review. Patient Education and Counseling, 48(2), $177-187$.

[5] Bowie C. R. and Harvey P. D. (2006) Administration and interpretation of the Trail Making Test. Nature protocols, 1(5), 2277-2281.

[6] Chung C., Dew K., Cole A., Zia J., Fogarty J., Kientz J. A., and Munson S. A. (2016) Boundary Negotiating Artifacts in Personal Informatics: Patient-Provider Collaboration with Patient-Generated Data. In Proceedings of the 19th ACM Conference on Computer-Supported Cooperative Work \& Social Computing, San Fransisco, February 2016 (New York: ACM), 770-786.

[7] Elmqvist N., Moere A. V., Jetter H., Cernea D., ReITERER H., and JANKUN-Kelly T. (2011) Fluid interaction for information visualization. Information Visualization 10(4): 327-340.

[8] FLECK R. and FITZPATRICK G. Reflecting on reflection: framing a design landscape. In Proceedings of OZCHI'10, Brisbane Australia, November 2010 (New York USA, ACM), 216-223.

[9] Frost J. and SMith B. (2003) Visualizing health: imagery in diabetes education. In Proceedings of the 2003 conference on Designing for user experience, San Fransisco USA, June 2003 (New York USA, ACM), 1-14.

[10] Greis M., Henze N., and Schmidt A. (2015) Predicting the Future: Towards Personal Simulation. In CHI'15: Workshop on 'Beyond Personal Informatics: Designing for Experiences of Data', Seoul Korea, April 2015 (New York USA: ACM Press), 1-14.

[11] Kvale S. (1996) Interviews: an introduction to qualitative research interviewing. (California: Sage, Thousand Oaks).

[12] Li I., DEY A. K., and ForLizZI J. (2010) A stage-based model of personal informatics systems. In Proceedings of the SIGCHI Conference on human factors in computing systems, Atlanta USA, (2010) (New York USA: ACM Press), 557-566.

[13] Li I., Dey A. K., and Forlizzi J. (2011) Understanding My Data, Myself: Supporting Self-Reflection with Ubicomp Technologies. In UbiComp'11 Proceedings of the 13th international conference on Ubiquitous computing, Beijing China, September 2011 (New York USA, ACM Press).

[14] Li I., MedynskiY Y., Froehlich J., and LARSEN J. (2012) Personal Informatics in Practice: Improving Quality of Life Through Data. In ACM SIGCHI Conference on Human Factors in Computing Systems, Austin Texas, May 2012 (New York USA: ACM Press), 2799-2802.

[15] Macleod H., TANG A., and Carpendale S. (2013) Personal informatics in chronic illness management. In Proc. of Graphics Interface'13, Regina Canada, May 2013 (Mississauga, Ontario: Canadian Information Processing Society), 149-156

[16] DeFanti T.A., and Brown M. D. (1991) Visualization in Scientific Computing. IEEE Computer Graphics and Applications 7(10): 69-69.
[17] KNOCHE, H., HALD, K., JøRGENSEN, H.R.M., and RichteR, D. 2016. Playing to (self-)rehabilitate: A month-long randomized control trial with brain lesion patients and a tablet game. Proc. of Pervasive Health'16, ACM, 61-68.

[18] NAyAK J. G., Hartzler A. L., Macleod L. C., IZARD J. P., DALKIN B. M., and GoRE J. L. (2016) Relevance of graph literacy in the development of patient-centered communication tools. Patient Education and Counseling 99(3): $448-454$.

[19] Rego P., Moreira P. M., and Reis L. P. (2010) Serious games for rehabilitation: A survey and a classification towards a taxonomy. In 5th Iberian Conference on Information Systems and Technologies, Santiago de Compostela Spain, June 2010 (Piscataway New Jersey: IEEE), 1-6.

[20] Rivera-Pelayo V., Zacharias V., Müller L, and Braun S. (2012) Applying Quantified Self Approaches to Support Reflective Learning. In Proc. of LAK '12, Vancouver Canada, April 2012 (New York USA, ACM), 111-114.

[21] Rosewilliam S., Roskell C. A., and Pandyan A. D. (2011) A systematic review and synthesis of the quantitative and qualitative evidence behind patient-centred goal setting in stroke rehabilitation. Clinical Rehabilitation 25(6): 501514 ,

[22] SCHÖN D. A. (1983) The reflective practitioner: how professionals think in action, (New York USA: Basic Books).

[23] Webb A. M., Linder R., Kerne A., Lupfer N., Qu Y., and POFFENBERGER B. Promoting reflection and interpretation in education: Curating rich bookmarks as information composition. In Proceedings of Creativity \& Cognition 13, Sydney Australia, June 2013 (New York USA: ACM Press).

[24] Wressle E., Eeg-Olofsson A., Marcusson J., and HENRIKSSON C. (2002) Improved client participation in the rehabilitation process using a client-centred goal formulation structure. Journal of Rehabilitation Medicine 34(1): 5-11.

[25] YIn R. K. (2011) Qualitative Research from Start to Finish. Guilford Press, New York, N.Y 